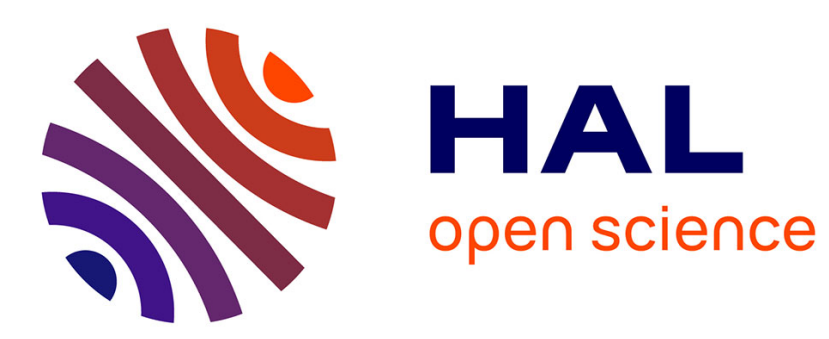

\title{
Advances in Internet of Things (IoT) in Manufacturing
} Rakshith Badarinath, Vittaldas V. Prabhu

\section{To cite this version:}

Rakshith Badarinath, Vittaldas V. Prabhu. Advances in Internet of Things (IoT) in Manufacturing. IFIP International Conference on Advances in Production Management Systems (APMS), Sep 2017, Hamburg, Germany. pp.111-118, 10.1007/978-3-319-66923-6_13 . hal-01666162

\section{HAL Id: hal-01666162 \\ https://hal.inria.fr/hal-01666162}

Submitted on 18 Dec 2017

HAL is a multi-disciplinary open access archive for the deposit and dissemination of scientific research documents, whether they are published or not. The documents may come from teaching and research institutions in France or abroad, or from public or private research centers.
L'archive ouverte pluridisciplinaire HAL, est destinée au dépôt et à la diffusion de documents scientifiques de niveau recherche, publiés ou non, émanant des établissements d'enseignement et de recherche français ou étrangers, des laboratoires publics ou privés. 


\title{
Advances in Internet of Things (IoT) in Manufacturing
}

\author{
Rakshith Badarinath $^{1}$ and Vittaldas V Prabhu ${ }^{1}$ \\ ${ }^{1}$ The Pennsylvania State University, University Park PA 16802, USA \\ rakshithb@psu.edu, vxp7@psu.edu
}

\begin{abstract}
As a promising technology with increased adoption in recent years, Internet of Things (IoT) realizes ubiquitous interconnection of physical devices through internet, opening doors for building powerful industrial applications by leveraging the advances in sensor technology and wireless networks. IoT technologies can be viewed as enablers for smart manufacturing and Industry 4.0. This review paper focuses on applications of IoT in manufacturing, which is also known as Industrial Internet of Things IIoT. To that end, technologies relevant to the application of IoT in manufacturing, such as wireless sensor networks (WSNs), smart sensors, big data analytics, and cloud computing are discussed. A service oriented architecture (SOA) based four-layer model for realizing IoT applications in manufacturing is proposed. Finally, a review of the state of art of IoT applications in manufacturing including shop floor automation, predictive maintenance, energy aware manufacturing, and smart workers is presented with relevant industry use cases.
\end{abstract}

Keywords: Industrial Internet of Things (IIoT), Big Data Analytics, Wireless Sensor Networks (WSN), Predictive Maintenance, Energy Aware Manufacturing, SOA, Smart Manufacturing, Industry 4.0

\section{$1 \quad$ Introduction}

\subsection{Understanding Internet of Things}

Internet of Things (IoT) can be defined as an ecosystem of interconnected, uniquely identifiable physical devices and software components having an ability to exchange data with other devices over a network with limited human intervention. The internet of things Global Standards Initiative (IoT-GSI) defines IoT as "a global infrastructure for the information society, enabling advanced services by interconnecting (physical and virtual) things based on existing and evolving interoperable information and communication technologies"[1]. One can visualize IoT as a seamless interconnection of the physical world and cyber worlds, with a pervasive presence around us [2]. IoT technologies provide means for physical objects to be monitored or controlled remotely over a network. This capability, coupled with ubiquitous interconnection of heterogeneous devices, opens doors for a new class of applications. The physical devices that can be interconnected vary from tiny electronics embedded into products to large automated buildings, vehicles, and everything in-between. An IoT application relies on a foundation created as a result of integration of sensors, actuators, and tracking devices 
such as RFID tags coupled with information and communication technologies, enabling cooperation among interconnected objects to achieve common goals.

There is an increased interest in using IoT in industries [3]. The three-year old Industrial Internet Consortium (IIC) comprises over 250 member companies [4]. Applications of IoT appear in a diverse set of industries including manufacturing, agriculture, traffic monitoring, food processing, health monitoring, and personal fitness. Additionally, there has been a sharp increase in the number of publications related to IoT. A search of Web of Science indicated that the number of publications in IoT increased from 0 to 6836 between 2006 and 2016. It is estimated that IoT will encompass 50 billion devices by 2020 [5].

This review focuses on identifying existing IoT technologies, architecture requirements, and applications specific to the manufacturing domain. In the current day global competitive environment, manufacturing companies are continuously striving to improve their competitiveness in terms of product price, time to market, efficiency of production processes, costs, etc. New technological paradigms like IoT play a crucial role in keeping companies competitive while meeting continuously changing customer needs. In fact, IoT has a great potential to transform the manufacturing sector [6] by creating novel business opportunities. IoT technologies enable integration of sensor and other electronics into various physical elements in a manufacturing plant where, with cooperative communication, entities can autonomously exchange information leading to processes that can govern themselves by taking intelligent decisions. This provides unprecedented opportunities for the manufacturing sector where resources can be monitored in real time, enabling higher safety and performance. This promising idea has led to several global initiatives such as "the Fourth Industrial Revolution" in German industry ( also called "Industry 4.0"), "Factory of the Future" in the European Union, and "Made in China 2025".

\section{Enabling Technologies for IoT Applications}

In this section, technologies which form the backbone of IoT applications in manufacturing are discussed.

\subsection{Wireless Sensor Networks (WSN)}

There are barriers to running cables on a factory floor because of potential safety hazards and associated costs. This is exacerbated as the distances between the connected points grow. WSNs can alleviate many of these barriers by offering numerous spatially distributed sensor nodes ${ }^{1}$ that are wirelessly connected through gateways to enable remote monitoring of physical or environmental conditions [7]. Table 1 summarizes currently available wireless communication technologies suitable for WSN [8], [9].

\footnotetext{
${ }^{1}$ Autonomy refers to devices equipped with sensors capable of self-recovery and making localized decisions.
} 
Table 1. Comparison of different communication technologies in IoT

\begin{tabular}{|c|c|c|c|c|}
\hline Technology & Range & Frequency & $\begin{array}{c}\text { Power } \\
\text { Consumption }\end{array}$ & Data Rate \\
\hline $\begin{array}{l}\text { NFC } \\
\text { (IEC 18000-3) }\end{array}$ & Up to $0.2 \mathrm{~m}$ & $13.56 \mathrm{MHz}$ & Low $(\sim 50 \mathrm{~mA})$ & Up to $424 \mathrm{~Kb} / \mathrm{s}$ \\
\hline $\begin{array}{l}\text { Bluetooth LE } \\
\text { (Bluetooth } 4.2 \\
\text { Core Spec) }\end{array}$ & Up to $100 \mathrm{~m}$ & $2.4 \mathrm{GHz}$ & $\begin{array}{l}\text { Low }(\sim 12.5 \\
\mathrm{mA})\end{array}$ & $\begin{array}{l}1 \mathrm{Mbps} \\
\text { (Smart/BLE) }\end{array}$ \\
\hline $\begin{array}{l}\text { Wi-Fi } \\
(\text { IEEE } \\
802.11 a / b / g)\end{array}$ & $\begin{array}{l}\text { Up to } 100 \mathrm{~m} \text { (can } \\
\text { be extended) }\end{array}$ & $2.4 \mathrm{GHZ} / 5 \mathrm{GHz}$ & $\begin{array}{l}\text { Medium/High } \\
(\sim 116 \mathrm{~mA})\end{array}$ & $\begin{array}{l}150-200 \\
\text { Mbps (down- } \\
\text { load); up to } 20 \\
\text { Mbps for up- } \\
\text { load }\end{array}$ \\
\hline WiMAX & Up to 30 miles & $2-11 \mathrm{GHz}$ & $\begin{array}{l}\text { High } \\
\text { (Depends on } \\
\text { range of cover- } \\
\text { age } \sim 200 \mathrm{~W} \text { ) }\end{array}$ & $\begin{array}{l}\text { Up to } 83 \mathrm{Mbps} \\
\text { (download); } \\
\text { up to } 46 \mathrm{Mbps} \\
\text { (upload) }\end{array}$ \\
\hline RFID & $\begin{array}{l}\text { Distance driven } \\
\text { by operating fre- } \\
\text { quency: up to } \\
200 \text { m for active } \\
\text { RFID }\end{array}$ & $\begin{array}{l}\mathrm{LF}: 125-134 \mathrm{kHz} \\
\mathrm{HF}: 13.56 \mathrm{MHz} \\
\mathrm{UHF}: 850-960 \mathrm{MHz}\end{array}$ & $\begin{array}{l}\text { Low } \\
\text { (Depends on } \\
\text { operating fre- } \\
\text { quency and tag } \\
\text { type) }\end{array}$ & $\begin{array}{l}\text { Up to } 640 \\
\text { Kbps }\end{array}$ \\
\hline $\begin{array}{l}\text { Cellular } \\
\text { (4G/LTE) }\end{array}$ & $\begin{array}{l}35 \mathrm{Km} \text { max for } \\
\text { GSM, } 200 \mathrm{Km} \\
\max \text { for HSPA }\end{array}$ & 1900/2100 MHZ & $\begin{array}{l}\text { High } \\
(\sim 184 \mathrm{~mA})\end{array}$ & $\begin{array}{l}3-10 \text { Mbps } \\
(\mathrm{LTE})\end{array}$ \\
\hline $\begin{array}{l}\text { Z Wave } \\
\text { (ITU-T G.9959) }\end{array}$ & $30 \mathrm{~m}$ & $900 \mathrm{MHz}$ & $\begin{array}{l}\text { Low } \\
(\sim 2.5 \mathrm{~mA})\end{array}$ & $\begin{array}{l}\text { Up to } 100 \\
\text { Kbps }\end{array}$ \\
\hline $\begin{array}{l}\text { ZigBee } \\
\text { (IEEE 802.15.4) }\end{array}$ & Up to $100 \mathrm{~m}$ & $2.4 \mathrm{GHz}$ & $\begin{array}{l}\text { Low }(< \\
10 \mathrm{~mW})\end{array}$ & $250 \mathrm{Kbps}$ \\
\hline $\begin{array}{l}\text { EnOcean } \\
\text { (IEC 14543-3) }\end{array}$ & $\begin{array}{l}\text { Up to } 300 \mathrm{~m} \\
\text { (outdoor) and } 30 \\
\mathrm{~m} \text { (indoor) }\end{array}$ & $\begin{array}{l}315 \mathrm{MHz}, 868 \mathrm{MHz} \text {, } \\
902 \mathrm{MHz}\end{array}$ & $\begin{array}{l}\text { Low } \\
\text { (Stand By: } 100 \\
\text { nA) }\end{array}$ & $\begin{array}{l}\text { Up to } 125 \\
\text { Kbps }\end{array}$ \\
\hline
\end{tabular}

A typical autonomous WSN node consists of radio, battery, microcontroller, analog circuit, and sensor interface [7]. As seen in Table 1, high data rate capability often results in power hungry devices. The selection of communication technology for manufacturing shop floor is largely influenced by the size of the plant, the number of physical assets that need to be monitored, and their location within the plant.

\subsection{Smart Sensors}

Sensors have evolved over the years from bulky dedicated devices to miniature multivariable sensing packages. Sensors are primary sources for any actionable data that can contribute to profitable business decisions. A smart sensor has localized computational capability to turn raw data into actions in real time, allowing fast responses and circumventing network latency issues. While some smart sensors have actuation capability, this function is mainly limited to dedicated hardware. A number of sensors are already 
being used the manufacturing domain, primarily to monitor machines and processes. A recent survey from PwC reveals that $35 \%$ of the manufacturing industry in US is already collecting and using data from smart sensors embedded in machines, to enhance operational efficiencies [10].

\subsection{Big Data Analytics}

Increased adoption of sensors to monitor physical assets such as machinery and end products leads to generation of massive amounts of raw data, estimated to be in the order of 1000 Exabytes (or 1 Zetabyte) annually per plant and expected to increase in coming years [11]. This massive amount of data, commonly referred to as big data, needs to be analyzed in a computationally efficient and timely manner for identifying trends and patterns among process parameters and outputs. Insights derived from such big data analytics is already changing decision-making in industry and a number of new ventures are targeting related business opportunities [10].

\subsection{Cloud Computing}

Cloud computing is changing the way industries do business by eliminating upfront cost for localized computing resources, and replacing this with lower-cost "pay-as-youuse" computing as a service over the Internet [12]. Moreover, cloud computing is dynamically scalable, which makes it an ideal platform for big data analytics where powerful computing resources may be used intermittently. Cloud computing's scalability, better resource utilization, and plug-and-play networks of heterogeneous devices have led to the emergence of "Cloud Manufacturing" [12]. Cloud manufacturing can be viewed as an approach where hardware and software functions are virtualized as a scalable, "elastic", virtual manufacturing enterprises that can be rapidly configured or shutdown. This can be expected to play an important role in realizing the full potential of the IoT paradigm.

\section{Architecture for IoT Applications in Manufacturing}

Service Oriented Architecture (SOA) consists of discrete services, which are composable to achieve a higher-level goal. SOA standards allow for a physical device's functionality to be offered as interoperable services independent of vendors, products, and technologies[13]. Since IoT is based on cooperative communication between heterogeneous devices to achieve a specific goal, SOA can be applied[14].

There has been a lot of research related to design of multi-layer SOA for IoT in general and also for specific domains like manufacturing. Gubbi et al. [15] proposed a cloud-based framework for generic IoT that includes three layers: network of things, cloud computing, and applications. Atzori et al. proposed a three-layer model for IoT consisting of an application layer, a network layer, and a sensing layer [2]. Lee et al. suggested a five-layer architecture model for cyber-physical manufacturing systems consisting of a sensing level for data acquisition; a data-to-information conversion level involving smart analytics for component health; a cyber level for cyber modelling and 
simulation; a cognition level for remote visualization; and a collaborative-diagnostics and configuration level which involves self-configuration and self-optimization based on learning [16]. Y. Zhang et al. proposed a real-time information capture and integration framework for IoT applications in manufacturing, comprising 3 layers: event sensing and capturing; manufacturing and data processing; and application services [17].

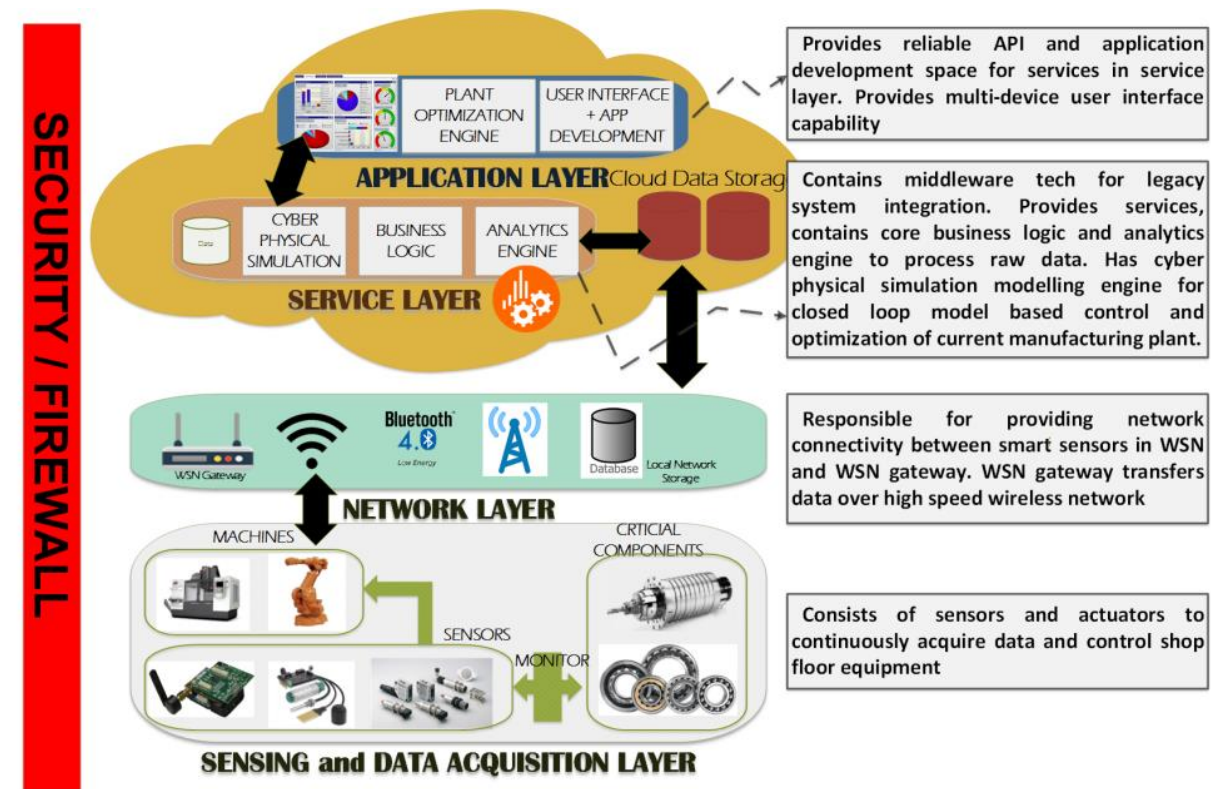

Fig. 1. Proposed SOA-based architecture for IoT applications in Manufacturing

The architectures proposed by Gubbie et al., Atzori et al., and Zhang et al. are functional architectures and are relatively compatible where IoT applications are present only at the top layer, whereas in the architecture proposed by Lee et al., each level has increasingly sophisticated IoT applications, making it a hybrid of functional and application architecture. In this paper, we build on these to propose a 4-Layer SOA for IoT applications in manufacturing including sensing and data acquisition, network, services, and applications layers as illustrated in Figure 1 along with the specific functionality for each layer. Note that our proposed architecture would be suitable for cloud manufacturing or non-cloud manufacturing. While no industrial implementation of the proposed architecture exists at present in its entirety, the individual elements of the architecture have been successfully realized in practice, and are presented in the next Section.

\section{$4 \quad$ IoT Applications in Manufacturing}

A: Real-time data collected from the shop floor can be used for designing an optimal production systems with automated work flows. Optimal decisions on production scheduling are driven by results of raw data processed through powerful analytics. Industry Example: At Siemens' electronics manufacturing plant in Amberg, Germany, 
machines and computers handle $75 \%$ of the operations autonomously, with roughly 1,000 automation controllers in operation along the production line. Parts being produced communicate with machines by means of a product codethat tells the machines their production requirements and which steps need to be taken next [18].

B: Analyzing real-time data on a machine's health in correlation with equipment process models can lead to predictive maintenance based on the remaining useful life estimation and automated condition-based maintenance strategies, leading to lower machine downtime and increased productivity. Industry Example: GE's digital windfarm (a fleet of IoT-enabled wind turbines) is monitored in real time and the collected data on rotor speed and weather conditions is used to build predictive-maintenance models using GE's Predix ${ }^{\mathrm{TM}}$ cloud-based analytics software. "Every day at $G E$ 's remote $M \& D$ Center in Atlanta, Georgia, GE collects more than 30,000 operating hours of data from a fleet of more than 1,500 assets. Insights drawn from this volume of power generation big data have translated into total customer savings estimated at $\$ 70$ million in 2014"[19].

C: The manufacturing sector typically accounts for one-third of the global energy demand [20]. An IoT application allows for continuous measurement of energy consumption of physical assets on the shop floor. This, when correlated with production plans and states of assets, can lead to dynamic, closed-loop energy regulation algorithms to intelligently regulate assets to the low-energy states, resulting in energy savings while maintaining the throughput level (despite the ramp-up time from a low-energy state to an operational state). This is not only applicable to manufacturing equipment, but also for plant HVAC and lighting systems. Industry Example: At BMW's Spartanburg (SC, USA) production plant, IoT-based smart energy meters constantly measure energy consumption of the facility to predict and identify sources of abnormal or high energy consumption. This led to total energy savings of over $100,000 \mathrm{kWh}$ in the first year, which is estimated to correspond to total energy cost savings of about 25 million euros over next 10 years. Additionally, the system can also identify imminent breakdowns of equipment by monitoring energy usage. For example, if a piece of equipment is using more energy without any change in production parameters, it is likely a result of malfunction or wear and tear of its components[21].

D: There is a new trend in applying wearable technology to enhance productivity and workforce safety. Industry Examples: Thyssenkrupp, AG has more than 24,000 service technicians who use mixed-reality devices in elevator service operations. Hands-free remote holographic guidance reduces the average length of service calls by up to four times [22]. Intel and Honeywell have collaborated to develop a system of wearable sensors that monitor a worker's breathing, heart rate, posture, and toxic-gas exposure. Data from the sensors is analyzed and displayed on cloud-based dashboards, helping managers better anticipate unsafe working conditions [23].

Mapping the application B to our four-layer SOA model, smart sensors in the sensing and data-acquisition layer continuously monitor the electronic signature of components of a windmill such as rotor bearings, rotor blades, gearbox, etc. This data is transmitted 
in real time to the service layer through WSNs in the network layer. The service layer may be cloud-based or on-premises proprietary software having analytics capability to sift through the data and detect any anomalies or trends that are indicative of component degradation by comparing the data with inputs from analytical reliability component models. These trends, along with estimated remaining useful life of the component derived by comparison to component reliability models, are presented on user dashboards in the application layer. This can be used to automatically trigger preventive maintenance or to alert service managers to schedule maintenance of affected components, helping to avoid unexpected downtime due to breakdowns and failures. Next, application $\mathrm{D}$ comes into action, where service technicians use mixed-reality wearable technology devices to efficiently identify the component and service it.

\section{Conclusions and Challenges}

This paper reviewed the main enabling technologies for IoT: wireless sensor networks, smart sensors, big data analytics, and cloud computing. Based on prior architectures for such systems, a new SOA-based four-layer architecture was proposed. Successful IoT applications in manufacturing were reviewed and mapped to the proposed four-layer architecture. Integration of smart-sensing and actuation capabilities on equipment with cyber space offers prospects of new levels of productivity, and could become an important dimension of competition in the future. A few of the current IoT applications in manufacturing used cloud computing, but most did not. There are several research challenges limiting widespread adoption IoT applications in manufacturing: (1) Data security/privacy concerns: opening up vast number of connected devices to the cloud offers multiple points of entry for an attacker; (2) Integration of legacy equipment often needs dedicated patches and may not be always feasible; (3) Need for open standards: Standards help improve interoperability of different systems resulting in sustainable functioning of the system and increased adoption; and (4) Technological barriers: manufacturing applications that require real-time feedback control may not be able to tolerate the data transfer latency that can arise with IoT. Other challenges include the high cost of sensor instrumentation and legal issues of data ownership. Overall, emerging IoT applications in manufacturing open new research challenges that require technology standards and solutions that can harness large streams of real-time data to optimize operations across the entire manufacturing life cycle.

\section{References}

[1] Internet of Things Global Standards Initiative, "Internet of Things Global Standards Initiative," 2015. Available: http://www.itu.int/en/ITU-T/gsi/iot/Pages/default.aspx.

[2] L. Atzori, A. Lera, and G. Morabito, "Internet of Things: A Survey," Comput. Networks, vol. 54, no. 15, pp. 2787-2805, 2010.

[3] Y. Li, M. Hou, H. Liu, and Y. Liu, "Towards a theoretical framework of strategic decision, supporting capability and information sharing under the context of Internet of Things," Inf. Technol. Manag., vol. 13, no. 4, pp. 205-216, 2012.

[4] "Current Members | Industrial Internet Consortium." [Online]. Available: 
http://www.iiconsortium.org/members.htm. [Accessed: 25-Apr-2017].

[5] D. Evans, "The Internet of Things - How the Next Evolution of the Internet is Changing Everything," CISCO white Pap., no. April, pp. 1-11, 2011.

[6] Z. Bi, L. Da Xu, and C. Wang, "Internet of things for enterprise systems of modern manufacturing," IEEE Trans. Ind. Informatics, vol. 10, no. 2, pp. 1537-1546, 2014.

[7] "What Is a Wireless Sensor Network? - National Instruments," 2016. [Online]. Available: http://www.ni.com/white-paper/7142/en/. [Accessed: 30-Mar-2017].

[8] Azamuddin Bin Ab Rahman, "Comparison of Internet of Things (IoT) Data Link Protocols," 2015.

[9] "IoT Technology 2017 Overview Guide on Protocols, Software, Hardware and Network Trends."Available: http://postscapes2.webhook.org/internet-of-things-technologies.

[10] PwC, "The Internet of Things: What It Means for US manufacturing," 2015.

[11] D. Mourtzis, E. Vlachou, and N. Milas, "Industrial Big Data as a Result of IoT Adoption in Manufacturing," in Procedia CIRP, 2016, vol. 55, pp. 290-295.

[12] X. Xu, "From cloud computing to cloud manufacturing," 2011.

[13] N. Ivezic, B. Kulvatunyou, and V. Srinivasan, "On Architecting and Composing Through-life Engineering Information Services to Enable Smart Manufacturing," Procedia CIRP, vol. 22, pp. 45-52, 2014.

[14] B. (Serm) Kulvatunyou, N. Ivezic, and V. Srinivasan, "On Architecting and Composing Engineering Information Services to Enable Smart Manufacturing," J. Comput. Inf. Sci. Eng., vol. 16, no. 3, p. 31002, Aug. 2016.

[15] J. Gubbi, R. Buyya, S. Marusic, and M. Palaniswami, "Internet of Things (IoT): A vision, architectural elements, and future directions," Futur. Gener. Comput. Syst., vol. 29, no. 7, pp. 1645-1660, 2013.

[16] J. Lee, B. Bagheri, and H. A. Kao, “A Cyber-Physical Systems architecture for Industry 4.0-based manufacturing systems," Manuf. Lett., vol. 3, pp. 18-23, 2015.

[17] Y. Zhang, G. Zhang, J. Wang, S. Sun, S. Si, and T. Yang, "Real-time information capturing and integration framework of the internet of manufacturing things," Int. J. Comput. Integr. Manuf., vol. 3052, no. December, pp. 1-12, 2014.

[18] K. O'Marah, “The Internet of Things Will Make Manufacturing Smarter," 2015. [Online]. Available: http://www.industryweek.com/manufacturing-smarter.

[19] http://www.windpowerengineering.com/featured/business-news-projects/ge-revealsvalue-of-connected-utility-with-the-predix-enabled-digital-power-plant/.

[20] S. Karnouskos, A. W. Colombo, J. L. M. Lastra, and C. Popescu, "Towards the energy efficient future factory," in IEEE International Conference on Industrial Informatics (INDIN), 2009, pp. 367-371.

[21] https://www.press.bmwgroup.com/global/article/detail/T0195345EN/industry-4-0:intelligent-energy-data-management-for-sustainable-production?

[22] https://www.thyssenkrupp.com/en/newsroom/press-releases/press-release114208.html.

[23] https://www.honeywell.com/newsroom/pressreleases/2015/11/honeywell-and-inteldemonstrate-prototype-of-wearable-iot-connected-safety-solutions-for-industrialworkers-and-first-responders. [Accessed: 04-Apr-2017]. 